\title{
Mechanism design and implementation theoretic perspective for interference coupled wireless systems
}

\author{
Holger Boche ${ }^{1 *}$, Siddharth Naik ${ }^{2}$ and Tansu Alpcan ${ }^{3}$
}

\begin{abstract}
This article investigates the properties of social choice functions (SCFs) that represent resource allocation strategies for interference coupled wireless systems. The resources can be physical layer parameters such as power vectors or spatial streams. Strategy proofness and efficiency properties of SCFs are used to capture the properties of non-manipulability and Pareto optimality of resource allocation strategies, respectively. This article introduces and investigates the concepts of (strong) intuitive fairness and non-participation in interference coupled systems. The analysis indicates certain inherent limitations when designing strategy proof and efficient resource allocation strategies, if additional desirable and intuitive properties are imposed. These restrictions are investigated in an analytical mechanism design framework for interference coupled wireless systems. The article also investigates the permissible SCFs, which can be implemented by a mechanism in either Nash equilibrium or dominant strategy for utility functions representing interference coupled wireless systems. Among other results, it is shown that a strategy proof and efficient resource allocation strategy cannot simultaneously satisfy continuity and the often encountered property of non-participation.
\end{abstract}

\section{Introduction}

From the evolution of wireless infrastructure from second generation to third generation, there has been a gradual transition from voice centric to data centric applications. Many of these applications are quality of service (QoS) based. A QoS application typically requires users to report their channel qualities to a central controller. The vendors manufacturing end user equipment have an incentive to report a higher channel quality, than the true channel quality experienced by the user. Such a misrepresentation of the channel quality is motivated by the vendor's intention of over provisioning for its users. There can be other instances, where the users have an incentive to misrepresent their measured channel quality or interference temperature. The result of solving a resource allocation problem with misrepresented utilities is that the outcome might not always be the one desired by the central controller, e.g., base station, operator. Such a misrepresentation of utilities can have an undesirable effect on the

\footnotetext{
*Correspondence: boche@tum.de

1 Department of Theoretical Information Technology, Technical University of Munich, Arcisstrasse 21, 80331 Munich, Germany

Full list of author information is available at the end of the article
}

resource allocation. Being in a position to tackle such a misrepresentation helps in better formulation of the optimization problem for radio resource allocation in wireless networks.

Expecting the resource allocation strategy to be strategy proof could be one possible solution to the central controller's dilemma of solving an optimization problem with misrepresented utilities. Much of previous strategy proofness literature in wireless network has been motivated from the perspective, that the users might have a motivation and ability to misrepresent their utilities (refer to the networks-related literature in "Literature survey" section).

We utilize the social choice function (SCF) to represent resource allocation strategies in interference coupled wireless systems. The goals of the designed resource allocation strategies can be viewed in terms of social choice, which is simply an aggregation of the preferences of the different users toward a single joint decision. The difference of interest between the operator and users is one example in networks, where the theory of mechanism design can be utilized.

\section{Springer}

(c) 2012 Boche et al. : licensee Springer. This is an Open Access article distributed under the terms of the Creative Commons Attribution License (http://creativecommons.org/licenses/by/2.0), which permits unrestricted use, distribution, and reproduction in any medium, provided the original work is properly cited. 
Mechanism design attempts implementing desired social choices in a strategic setting, assuming that the different members of society act rationally in a game theoretic sense [1].

We utilize an axiomatic framework for SCFs (discussed in detail in "SCFs" section). An SCF represents a resource allocation strategy in interference coupled wireless systems. In our abstraction, if an SCF satisfies a particular axiom, then the resource allocation strategy is said to satisfy the property corresponding to the axiom. We capture the non-manipulation of the resource allocation strategy by the property of strategy proofness of the SCF. An example of a strategy proof SCF is the second price auction (Vickrey Clarke Groves auction). Pareto optimality of the resource allocation strategy is captured by the property of efficiency. This article studies such and certain other desirable properties of SCFs representing resource allocation strategies.

We consider resource sets beyond pure exchange economies. The only constraint on our resource sets is that they satisfy the signal-to-interference plus noise ratio (SINR)-based utility (SBU) function framework (see "Utility modeling" section). This article provides certain new insights on a particular class of strategy proof and efficient resource allocation strategies and has the following main contributions (see "Analysis: properties of resource allocation strategies" section):

1 We introduce the property of intuitive fairness (see "SCFs" section). Intuitive fairness implies that if a particular user scales down its demand for utility, then the other users must obtain the same or better utility. For strong intuitive fairness, the users can choose from a family of utility functions.

(a) A strategy proof and efficient resource allocation strategy, which satisfies the property of intuitive fairness (see Definition 6) is robust to a particular user's scaling down of the utility, when the utilities of all other users are fixed.

(b) A strategy proof and efficient resource allocation strategy, satisfying either intuitive fairness (see Definition 6) or strong intuitive fairness (see Definition 7), can be altered only if two or more users change their utilities, i.e., the resource allocation strategy is robust to the change in utilities of any singular user.

(c) If a strategy proof and efficient resource allocation strategy is not constant with respect to the utility of a user $k$, then another user $j(j \neq k)$ experiences a measurable decrease in its performance, even if this other user $j$ 's utility function is fixed.
2 We introduce the property of non-participation, which says that if a particular user does not demand any utility, then it obtains no resource.

A strategy proof and efficient resource allocation strategy for interference coupled systems cannot simultaneously satisfy continuity and the property of non-participation. Continuity is a desirable property of resource allocation strategies for designing practical algorithms and for mathematical tractability. Hence, this result proves to be an impossibility result, i.e., a strategy proof, efficient, and non-participation resource allocation strategies are discontinuous.

3 Let a mechanism implement an SCF in Nash equilibrium. Then, there exists a "point" in the set of physical layer resources, such that the SCF chooses this point for all possible utility functions in the family of SBU functions. A similar result can be proved for dominant strategy implementation.

4. A resource allocation strategy is strategy proof, if and only if the SINR function $\gamma_{k}$ for a particular user $k$ is a constant function, independent of its own utility $u_{k}$. The constant mentioned in the previous sentence depends on the utility functions of the other users.

There has been a significant amount of economic literature on this topic. We give a brief overview of this literature in "Literature survey" section. It can be observed from the literature that previous work in networks and communication theory has typically focused on the design of strategy proof resource allocation strategies for particular wireless or communication systems. This article characterizes certain boundaries while designing strategy proof and efficient resource allocation strategies, when combined with certain desirable and intuitive properties. We investigate these contradictions in the framework of interference coupled wireless systems and an analytical framework of mechanism design, described in the following section.

\section{Analytical framework}

In this article, we shall investigate the case of interference coupled wireless systems, beyond pure exchange economies. Before we begin to describe our system model and present the relevant definitions, we provide certain notational conventions used in the article in the next section.

\section{Preliminaries and notation}

Matrices and vectors are denoted by bold capital letters and bold lowercase letters, respectively. Let $\boldsymbol{y}$ be a vector, then $y_{l}=[\boldsymbol{y}]_{l}$ is the $l$ th component. Let $\boldsymbol{y}_{-l}$ denote the vector $y$ without the $l$ th component. Likewise $G_{m n}=$ $[\boldsymbol{G}]_{m n}$ is a component of the matrix $\boldsymbol{G}$. The notation 
$\boldsymbol{y} \geq 0$ implies that $y_{l} \geq 0$ for all components $l . x \supsetneqq \boldsymbol{y}$ implies component-wise inequality with strict inequality for at least one component. Similar definitions hold for the reverse directions. $\boldsymbol{x} \neq \boldsymbol{y}$ implies that the vector differs in at least one component. Let $\mathcal{Y}$ denote a set of vectors and denote a family (class) of functions or tuples. We use $y_{k}$ for indexing of individual components of vectors and individual components of tuples of functions, where $y_{k} \in \mathcal{Y}_{k}$. Let $\mathcal{Y}^{K}=: \times_{k \in \mathcal{K}} \mathcal{Y}_{k}$ unless otherwise specified. The set of non-negative real numbers and positive real numbers are denoted as $\mathbb{R}_{+}$and as $\mathbb{R}_{++}$, respectively.

\section{Interference coupled wireless systems}

In a wireless system, the users' utilities can strongly depend on the underlying physical layer. An important measure for the link performance in cellular and ad hoc wireless networks is the SINR. SINR is also utilized as a performance indicator in the standardization community. Hence, results pertaining to such a model could help investigate the limitations of SINR-based communication systems. Consider $K$ users with transmit powers $\boldsymbol{p}=\left[p_{1}, \ldots, p_{K}\right]^{T}$ and $\mathcal{K}:=\{1, \ldots, K\}$. The noise power at each receiver is $\sigma^{2}$. Hence, the SINR at each receiver depends on the extended power vector

$$
\underline{\boldsymbol{p}}=\left[\begin{array}{l}
\boldsymbol{p} \\
\sigma^{2}
\end{array}\right]=\left[p_{1}, \ldots, p_{K}, \sigma^{2}\right]^{T} .
$$

The resulting SINR of user $k$ is

$$
\operatorname{SINR}_{k}(\underline{\boldsymbol{p}})=\frac{p_{k}}{\mathcal{I}_{k}(\underline{\boldsymbol{p}})}=\gamma_{k}(\underline{\boldsymbol{p}})
$$

where $\mathcal{I}_{k}$ is the interference (plus noise) as a function of $\boldsymbol{p}$. In order to model interference coupling, we shall fol$\overline{\text { low }}$ the axiomatic approach proposed in $[2,3]$. The general interference functions possess the properties of conditional positivity, scale invariance, and monotonicity with respect to the power component and strict monotonicity with respect to the noise component. For further details, kindly refer to the Appendix.

\section{Utility modeling}

In mathematical economics, the modeling of users' utilities is an initial step toward characterizing the preferences of the users and in turn utilizing the framework of mechanism design and implementation theory. In our system model, each user can choose its own utility function. For a user, announcing its true utilities to the operator might not be in its best interest, i.e., the users can choose to reveal a utility function, which differs from their true utility functions, so as to obtain more utility.

Generally, it is not possible to accurately communicate a non-parametric utility function in an Euclidean space.
However, for the purpose of obtaining certain initial intuition on the topic we have not concerned ourselves with this issue. For a practical implementation we can utilize approximations, e.g., a parametrization, where one could transmit a scalar and choose a function from a look up table based on the transmitted scalar or transmission of a finite number of scalars (based on the system constraints, e.g., bandwidth, time duration of block fading, etc.), which represent coefficients of a polynomial utilized to approximate the utility function. Scalar parameterized mechanisms have been discussed in [4].

We are particularly interested in analyzing the class of utility functions, which are functions of the SINR, given by (2). The utility functions, which shall be introduced in Definition 1, are motivated based on the below two factors.

- Users in a wireless system are coupled by interference.

- Performance indicators in wireless systems are influenced by physical layer parameters.

Definition 1. For user $k, u_{k}$ is said to be an SBU function, if there exists a strictly monotonic, increasing and continuous function $q$ and an interference function $\mathcal{I}_{k}$ such that

$$
u_{k}(\underline{\boldsymbol{p}})=q\left(\frac{p_{k}}{\mathcal{I}_{k}(\underline{\boldsymbol{p}})}\right)=q\left(\gamma_{k}(\underline{\boldsymbol{p}})\right)
$$

Remark 1. Let $\boldsymbol{u}=\left[u_{1}, \ldots, u_{K}\right] \in \mathcal{U}^{K}$, where $\mathcal{U}^{K}$ is the family of SBU functions for $K$ users.

In this article, "utility" can represent certain arbitrary performance measures, which depend on the SINR by a strictly monotonic, increasing, and continuous function $q$ defined on $\mathbb{R}_{+}$. The utility of user $k$ is

$$
u_{k}(\underline{\boldsymbol{p}})=q\left(\gamma_{k}(\underline{\boldsymbol{p}})\right), \quad k \in \mathcal{K} .
$$

An example of the above case is capacity: $q(x)=\log (1+$ $x)$ and effective bandwidth $q(x)=x /(1+x)$ [5]. The same theory can be developed for strictly monotonic, decreasing, and continuous functions $\hat{q}$. For the following performance indicators, we would like to minimize the objective function, e.g., mean square error : $\hat{q}(x)=1 /(1+$ $x)$, BER: $\hat{q}(x)=Q(\sqrt{x})$, and high-SNR approximation of $\operatorname{BER} \hat{q}(x)=x^{-\alpha}$ with diversity order $\alpha$.

\section{Analytical framework: SCFs and mechanism design}

In this section, we review certain mechanism design and implementation theoretic notation [6], in the context of interference coupled systems. We assume that the number of users $K \geq 2$. Let $\mathcal{R}$ be an arbitrary set of outcomes at the physical layer. Let $\mathcal{R}:=\times_{k \in \mathcal{K}} \mathbf{R}_{k}$ and $r_{k} \in$ $\mathbf{R}_{k}$. Resources at the physical layer are power, antenna weights, spatial streams, etc. A combination of these could also be considered as resources and modeled by our framework. 
Example 1. Consider a SIMO uplink scenario with a total power constraint or a MISO downlink scenario with a total power constraint $P_{\text {total }}$ and beamforming vectors for the users being $\omega_{k}$, with $k \in \mathcal{K}$. The set of resources $\mathcal{R}$ can be represented in this scenario as follows.

$$
\begin{aligned}
\mathcal{R} & =\left\{\left(\boldsymbol{p}, \boldsymbol{\omega}_{1}, \ldots, \boldsymbol{\omega}_{K}\right) \mid \boldsymbol{p} \geq \mathbf{0}, \sum_{k \in \mathcal{K}} p_{k} \leq P_{\text {total }},\left\|\omega_{1}\right\|_{2}\right. \\
& \left.=\cdots=\left\|\omega_{K}\right\|_{2}=1\right\}
\end{aligned}
$$

Let $r=\left[r_{1}, \ldots, r_{K}\right]$. A result is said to be presented for the case of pure exchange economies, if the resource set is defined as follows:

$$
\mathcal{R}=\left\{\boldsymbol{r} \in \mathbb{R}_{+}^{K} \mid \sum_{k \in \mathcal{K}} r_{k} \leq C, C \in \mathbb{R}_{+}\right\} .
$$

As mentioned in the "Introduction" section, in this article we analyze the case of interference coupled wireless systems, beyond pure exchange economies.

\section{SCFs}

Each user $k$ has a preference relation defined over the set of outcomes $\mathcal{R}$, which admits a numerical representation $u_{k}: \mathcal{R} \mapsto \mathbb{R}_{+}$

Remark 2. As has been observed earlier in this article, the set of outcomes $\mathcal{R}$ is the set of resources, which could be the set of power $\mathcal{P}$. In this special case, where power allocation is an outcome, we have that the particular power vector $\boldsymbol{p} \in \mathcal{P}$.

Example 2. Consider the case, when the users report their utility functions to a central controller, e.g., base station. Based on a system objective (an SCF), the base station allocates resources to the users. Based on the allocated resources, the users derive their own performance, e.g., QoS level.

Different users in a wireless system could have different preferences depending on their resource allocation strategy. We shall utilize the SCF to characterize resource allocation strategies. If a particular property (axiom) is satisfied by the SCF, then the corresponding property is satisfied by the resource allocation strategy, i.e., we utilize certain properties (axioms) to emulate desirable properties of resource allocation strategies.

An SCF aggregates the preferences of all the users into a social choice for the entire system, i.e., the resource allocation strategy.

Definition 2. An SCF is a function $f: \mathcal{U}^{K} \mapsto \mathcal{R}$ (i.e., $f$ is an injective function) that associates with every $\boldsymbol{u} \in \mathcal{U}^{K}$ a unique outcome $f(\boldsymbol{u})$ in $\mathcal{R}$.

We now clarify that what we mean by a strategy $K$-tuple and a strategy set. A strategy is a complete contingent plan or decision rule that says what a user will do at each of its information sets. Let $\mathcal{S}_{k}$ be the strategy set of a user $k \in \mathcal{K}$ and $\mathcal{S}^{K}:=\times_{k \in \mathcal{K}} \mathcal{S}_{k}$ be the strategy set of the set of users $\mathcal{K}$. We now present certain well-known desired properties of SCFs. We shall revisit strategy $K$-tuples and strategy sets, when we deal with mechanisms and implementation theoretic concepts in "Mechanism design and implementation theoretic concepts" section.

\section{Review of extant properties of SCFs}

We formalize certain desirable properties of resource allocation strategies by means of an axiomatic framework for SCFs to capture these properties. In this article, we are interested in exploring the interplay between the axiomatic framework and the implementation of resource allocation strategies.

Example 3. Consider for two users an SCF $f$ (see Equation 7). We analyze the case for linear interference functions and for a total power constraint $P_{\text {total }}$. Then we have that $\gamma_{1}(\underline{\boldsymbol{p}})=\frac{p_{1}}{v_{12} p_{2}+\sigma^{2}}$ and $\gamma_{2}(\underline{\boldsymbol{p}})=\frac{p_{2}}{v_{21} p_{1}+\sigma^{2}}$, where $v_{12}$ and $v_{21}$ are the normalized coupling between user 1 and 2 . Let the utility sets for the users be as follows:

$$
\mathcal{U}^{(1)}=\left\{\omega_{1} \log \left(\gamma_{1}(\underline{\boldsymbol{p}})\right)\right\} ; \quad \mathcal{U}^{(2)}=\left\{\omega_{2} \log \left(\gamma_{2}(\underline{\boldsymbol{p}})\right)\right\},
$$

where $\left[\omega_{1}, \omega_{2}\right]=\boldsymbol{\omega}>\mathbf{0}$. Let us choose the following $f$ :

$$
f(\boldsymbol{\omega})=\underset{s: e^{s_{1}}+e^{s_{2}} \leq P_{\text {total }}}{\operatorname{argmax}}\left(\omega_{1} \log \gamma_{1}\left(e^{\boldsymbol{s}}\right)+\omega_{2} \log \gamma_{2}\left(e^{\boldsymbol{s}}\right)\right),
$$

where $\boldsymbol{s}=\left[s_{1}, s_{2}\right], \boldsymbol{p}=e^{s}$ such that $p_{k} \in \boldsymbol{p}, p_{k}=e^{s_{k}}$ and $P_{\text {total }}$ is the total power constraint on the system for 2 users (see Figure 1b). The function $\omega_{1} \log \gamma_{1}\left(e^{s}\right)+\omega_{2} \log \gamma_{2}\left(e^{s}\right)$ is strictly convex and bounded. Therefore, there exists a unique optimizer, i.e., the function $f$ is a well-defined SCF. From (7) we can see that a user has an incentive to misrepresent its utility function.

By misrepresenting its utility function, a user can manipulate the outcome of a resource allocation strategy. Avoiding such behavior is a desired property from the perspective of an operator or a regulator. The property, that a particular resource allocation strategy is nonmanipulable, is emulated by the $\operatorname{SCF} f$ satisfying the property strategy proofness. The following two definitions can also be found in [7].

Definition 3. An SCF $f$ is said to be strategy proof, if for all users $k \in \mathcal{K}$ and for all utility functions $u_{k}, \hat{u}_{k} \in \mathcal{U}, \forall \hat{\boldsymbol{u}}_{-k} \in$ $\mathcal{U}^{K-1}$, we have that $u_{k}\left(f\left(u_{k}, \hat{\boldsymbol{u}}_{-k}\right)\right) \geq u_{k}\left(f\left(\hat{u}_{k}, \hat{\boldsymbol{u}}_{-k}\right)\right)$.

An SCF is said to be strategy proof if the users have no incentive to misrepresent their utilities to the central controller.

Definition 4. An SCF $f$ is efficient if $\forall \boldsymbol{u} \in \mathcal{U}^{K}$,

1. there is no $\boldsymbol{r} \in \mathcal{R}$ such that $u_{k}(\boldsymbol{r}) \geq u_{k}(f(\boldsymbol{u}))$ for all users $k \in \mathcal{K}$, and

2. $u_{k}(\boldsymbol{r})>u_{k}(f(\boldsymbol{u}))$ for some user $k \in \mathcal{K}$.

Efficiency from the point of view of wireless communication (physical layer perspective) of the resource allocation 

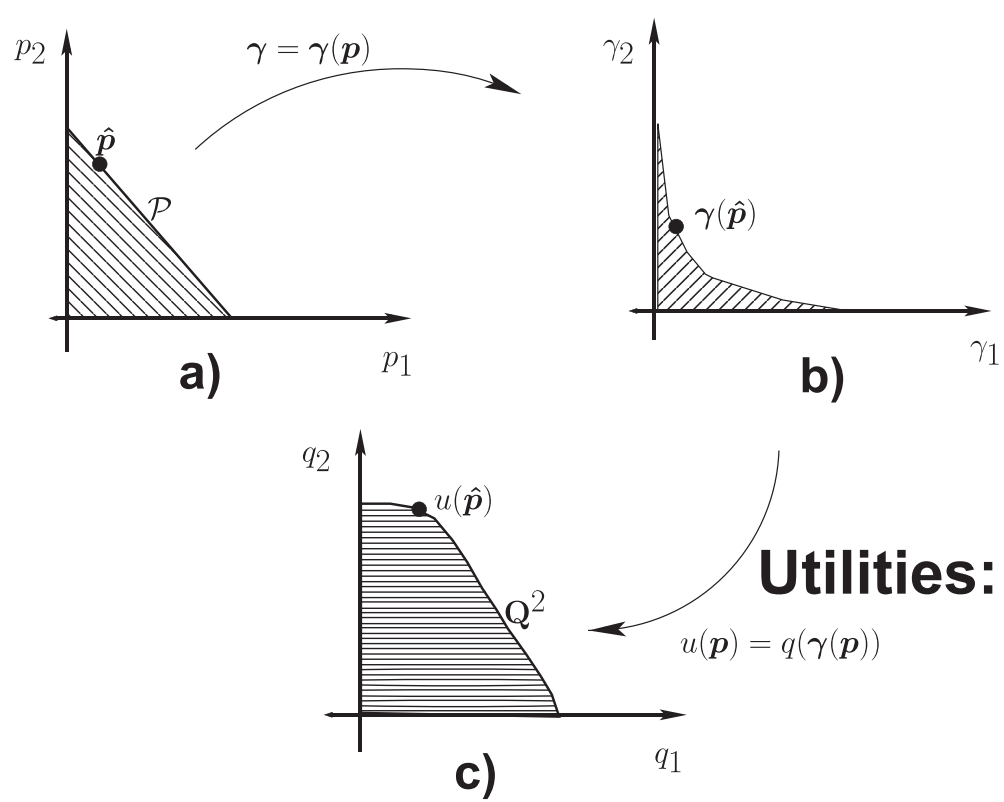

Figure 1 Depiction of a the set of resources $\mathcal{P}$ and the QoS set $\mathcal{Q}^{2}$ for the case of $\mathbf{2}$ users in a wireless system. (a) Set of resources for two users. In this case, the set of powers permitted by the power constraints for the two users. (b) SINR region corresponding to the set of powers, with the transformation $\boldsymbol{\gamma}=\boldsymbol{\gamma}(\boldsymbol{p})$. (c) QoS region after the transformation of the SINR region via the utility function mapping $u(\boldsymbol{p})=q(\boldsymbol{\gamma}(\boldsymbol{p}))$.

strategies implies choosing an operating point on the Pareto boundary of the feasible utility region [8].

Definition 5. The option set of a user $k \in \mathcal{K}$, given a utility function $(K-1)$-tuple $\boldsymbol{u}_{-k} \in \mathcal{U}^{K-1}$, is the set

$$
\mathbf{Q}_{k}\left(\boldsymbol{u}_{-k}\right)=\left\{\boldsymbol{r} \in \mathcal{R} \mid \exists u_{k} \in \mathcal{U} \text {, such that } f\left(u_{k}, \boldsymbol{u}_{-k}\right)=\boldsymbol{r}\right\}
$$

where $\boldsymbol{r}$ is a resource vector.

The option set $\mathbf{Q}_{k}$ is the set of resources for all the users, which user $k$ can influence with its utility function, given the utility function $(K-1)$-tuples $\boldsymbol{u}_{-k} \in \mathcal{U}^{K-1}$. The use of option sets has proved to be a useful technique in analyzing strategy proof SCFs [9]. The reader should bear in mind that option sets are relative to a given function on a given domain, even if this is not explicit in the notation. We shall now present certain new properties, which are quite natural from a wireless system perspective.

\section{Introduced properties of SCFs}

In this section, we introduce the properties of (strong) intuitive fairness and non-participation and connect them with certain well established concepts in literature.

Definition 6. An SCF $f$ is said to satisfy the property of intuitive fairness, if for all utility function $K$-tuples $\boldsymbol{u} \in$ $\mathcal{U}^{K}$, for all user $k \in \mathcal{K}$ we have that, for arbitrarily chosen $\left(u_{k}, \boldsymbol{u}_{-k}\right)$ and $0<\lambda<1, u_{k}\left(f\left(\lambda u_{j}, \boldsymbol{u}_{-j}\right)\right) \geq u_{k}\left(f\left(u_{j}, \boldsymbol{u}_{-j}\right)\right)$, $k \in \mathcal{K}, k \neq j$.

An SCF is said to be intuitive fair, if for all users $k \in \mathcal{K}$, we have the case, that if any user linearly scales down its utility, then the other users should either obtain the same or better utility as in the case, when the user had not scaled its utility. Definition 6 is similar to the axiom of population monotonicity (in the context of cooperative bargaining theory [10]) introduced under a different name in [11]. The axiom of population monotonicity states the following. Suppose a group of users $\mathcal{K}_{1}$ have arrived to play a particular resource allocation game. If the users $\mathcal{K}_{2} \backslash \mathcal{K}_{1}$ (with $\mathcal{K}_{1} \subset \mathcal{K}_{2}$ ) do not show up, let the set of users $\mathcal{K}_{1}$ reach a particular solution outcome. If the users $\mathcal{K}_{2} \backslash \mathcal{K}_{1}$ show up afterwards, resource allocation is carried out again and no user in $\mathcal{K}_{1}$ should be better off.

Now, we allow the user to possess the ability of not only scaling its utility function, but also choosing other utility functions altogether.

Definition 7. An SCF $f$ is said to satisfy the property of strong intuitive fairness, if for all users $k \in \mathcal{K}$, for all utility function $(K-1)$-tuples $\boldsymbol{u}_{-k} \in \mathcal{U}^{K-1}, u_{k}, \hat{u}_{k} \in \mathcal{U}$ and for $0 \leq \hat{u}_{k}(\boldsymbol{r}) \leq u_{k}(\boldsymbol{r})$ for all $\boldsymbol{r} \geq \mathbf{0}$, we have that

$$
u_{k}\left(f\left(\hat{u}_{j}, \boldsymbol{u}_{-j}\right)\right) \geq u_{k}\left(f\left(u_{j}, \boldsymbol{u}_{-j}\right)\right), \quad k \in \mathcal{K} \bigvee
$$

In the definition of strong intuitive fairness it can be seen that the utility function $\hat{u}_{k}$ is dominated by the utility function $u_{k}$, for all users $k \in \mathcal{K}$, for all resource vectors $\boldsymbol{r} \in \mathcal{R}$, and all utility function $(K-1)$-tuples $\boldsymbol{u}_{-k} \in \mathcal{U}^{K-1}$. Remark 3. The SCF $f$ presented in Example 3 satisfies the properties of efficiency and strong intuitive fairness.

The properties of intuitive fairness and strong intuitive fairness are somehow connected to the property of 
min-max fairness. For any resource allocation strategy providing QoS to the users we can associate a specific notion of fairness. The consideration of fairness notions has mainly been a wired network issue [12,13]. The most common fairness notion is min-max fairness. It represents an equilibrium associated with an ideal social system characterized by the fact that no user's QoS measure can be increased without decreasing an already lower user's QoS measure.

Example 4. In the framework of interference coupled systems the min-max fair power allocation solves the problem

$$
\min _{\boldsymbol{p} \in \mathcal{P}} \max _{k \in \mathcal{K}} \frac{q_{k}(\boldsymbol{p})}{q_{k}^{(\mathrm{req})}}=\min _{\boldsymbol{p} \in \mathcal{P}} \max _{k \in \mathcal{K}} \frac{f\left(\frac{[\boldsymbol{V} \boldsymbol{p}]_{k}}{p_{k}}\right)}{f\left(\frac{1}{\gamma_{k}^{(\text {req })}}\right)},
$$

where $q_{k}^{\text {req }}$ describes the QoS requirement of the $k$ th user and $\gamma_{k}^{\text {(req) }}$ the corresponding SINR threshold. $V$ is the link gain matrix for the interference coupled wireless system. Fortunately, in cellular wireless networks the intricacies associated with the so-called bottleneck connections are nonexistent. Under non-existing or equal QoS requirements the min-max fair power allocation equalizes all link QoS measures and represents the right eigen-vector of the interference matrix.

Comparing min-max fairness to intuitive fairness, we can see that if a particular user $k$ reduces its demand for utility, then there are more resources for the remaining users. Hence, another user $j \in \mathcal{K} \backslash k$ could increase its utility without decreasing the utility of a user $m \in \mathcal{K} \backslash\{j, k\}$.

We now discuss another property of resource allocation strategies, namely pointwise continuity. We say that the sequence of functions $\left\{\boldsymbol{u}^{(n)}\right\}_{n \in \mathbb{N}}, \boldsymbol{u}^{(n)} \in \mathcal{U}^{K}$ converges to $\boldsymbol{u} \in \mathcal{U}^{K}$, if for all constants $R_{\text {total }}>0$, we have that

$$
\lim _{n \rightarrow \infty} \max _{\boldsymbol{r} \geq \mathbf{0}, \sum_{k \in \mathcal{K}} r_{k} \leq R_{\text {total }}}\left\|\boldsymbol{u}^{(n)}(\boldsymbol{r})-\boldsymbol{u}(\boldsymbol{r})\right\|_{l^{1}}=0 .
$$

The sequence of utility functions $\left\{u_{k}^{(n)}\right\}_{n \in \mathbb{N}}$ are defined by their values, so the utility functions converge if their values converge. This reduces the convergence of realvalued functions to the convergence of real numbers. Such a convergence is called pointwise convergence. We are dealing with utility function $K$-tuples ( $K$-tuple of utility functions) as against utility functions. Hence, we use the $l-1$ norm. We would like at this point to remind the reader that we say that an SCF $f$ is continuous, if for all convergent sequences of utility $K$-tuples $\left\{\boldsymbol{u}^{(n)}\right\}_{n \in \mathbb{N}}$ the following expression holds:

$$
\lim _{n \rightarrow \infty}\left\|f\left(\boldsymbol{u}^{(n)}\right)-f(\boldsymbol{u})\right\|_{l^{1}}=0 .
$$

We now explain a very natural property, which is almost always satisfied for all resource allocation strategies occurring in interference coupled wireless systems. The property states that, if a particular user demands no utility, then the resource allocation strategy does not allocate any resource to this user.

Definition 8. An SCF $f$ is said to satisfy the property of non-participation, if for a given user $k \in \mathcal{K}$ and for all utility function $(K-1)$-tuples $\boldsymbol{u}_{-k} \in \mathcal{U}^{K-1}$, we have that

$$
f_{k}\left(0, \boldsymbol{u}_{-k}\right)=0 .
$$

Remark 4. In practical wireless networks it must be noted that if a user requires no utility, i.e., it demands no resources at a particular time instant, it still has to utilize some resources to report its utility function to the resource allocation agent (central controller). Hence, the property of non-participation though seemingly intuitive and harmless could lead to certain restrictions for resource allocation strategies, when it is expected to be satisfied with certain other properties. This will displayed in detail later in the proof of Theorem 3 (where the interplay of axioms of non-participation and continuity along with strategy-proofness and efficiency is brought to light).

Equipped with the suitable notations and framework, we present the results of our analysis in "Analysis: properties of resource allocation strategies" section.

\section{Mechanism design and implementation theoretic concepts}

In the previous section, we have seen that the SCF being used as a tool to capture certain desirable properties of resource allocation strategies in a wireless system. We shall now like to shift our focus to investigating the implementation aspects of resource allocation strategies in a wireless network. For this purpose, we shall utilize the theory of mechanism design and implementation theory. We begin by introducing the mechanism below.

Definition 9. A mechanism is a function $g: \mathcal{S}^{K} \mapsto \mathcal{R}$ that assigns to every strategy $K$-tuple $s \in \mathcal{S}^{K}$ a unique element $r \in \mathcal{R}$.

A mechanism is a procedure for determining outcomes. Who gets to choose the mechanism, i.e., who is mechanism designer depends on the scenario in question, e.g., base station, operator, regulator, etc.

Example 5. Consider an example, where the resources at the physical layer are only the powers of the users, i.e., $\mathcal{R}=\mathcal{P}$, with $\mathcal{P}$ the set of powers defined as follows: $\mathcal{P}=\left\{\boldsymbol{p} \mid \sum_{k \in \mathcal{K}} p_{k} \leq P_{\text {total }}\right\}$ and the utility function is defined by (4). With this scenario, Figure 1 displays the concepts of an $\operatorname{SCF} f$, the set of outcomes $\mathcal{P}$ and the set of utilities $\mathcal{U}^{K}$.

Let $g\left(\mathbf{S}_{k}, \boldsymbol{s}_{-k}\right)$ be the attainable set of user $k$ at $\boldsymbol{s}_{-k}$, i.e., the set of outcomes that user $k$ can induce when the other users select $\boldsymbol{s}_{-k}$. For $k \in \mathcal{K}, u_{k} \in \mathbf{U}_{k}$ and a resource vector $\boldsymbol{r} \in \mathcal{R}$, let $\mathbf{L}\left(\boldsymbol{r}, u_{k}\right)=\left\{\hat{\boldsymbol{r}} \in \mathcal{R} \mid u_{k}(\boldsymbol{r}) \geq u_{k}(\hat{\boldsymbol{r}})\right\}$ be the weak lower contour set of user $k$ with $u_{k}$ at resource vector $\boldsymbol{r}$.

Definition 10. Given a mechanism $g: \mathcal{S}^{K} \mapsto \mathcal{R}$, the strategy profile $\boldsymbol{s}^{*} \in \mathcal{S}^{K}$ is a Nash equilibrium of $g$ at 
$\boldsymbol{u} \in \mathcal{U}^{K}$, if and only if for all users $k \in \mathcal{K}$ and for all $(K-1)$-strategy tuples $\boldsymbol{s}_{-k} \in \mathbf{S}_{k}$ we have that

$$
u_{k}\left(g\left(s_{k}, s_{-k}^{*}\right)\right) \leq u_{k}\left(g\left(s_{k}^{*}, s_{-k}^{*}\right)\right)
$$

The Nash equilibrium of a mechanism can also be characterized in terms of the weak lower contour set as follows. Given a mechanism $g: \mathcal{S}^{K} \mapsto \mathcal{R}$, the strategy profile $\boldsymbol{s} \in \mathcal{S}^{K}$ is a Nash equilibrium of $g$ at $\boldsymbol{u} \in \mathcal{U}^{K}$ if for all $k \in \mathcal{K}$, $g\left(\mathbf{S}_{k}, \boldsymbol{s}_{-k}\right) \subseteq \mathbf{L}\left(g(\boldsymbol{s}), u_{k}\right)$. Let $N^{g}(\boldsymbol{u})$ be the set of Nash equilibria of the mechanism $g$ at utility function tuple $\boldsymbol{u}$. We now introduce the corresponding implementation theoretic concept of Nash equilibrium implementation.

Definition 11. The mechanism $g$ implements the SCF $f$ in Nash equilibrium, if for each utility function $K$-tuple $\boldsymbol{u} \in \mathcal{U}^{K}$, the following condition is fulfilled.

- For any strategy K-tuple $\boldsymbol{s} \in N^{g}(\boldsymbol{u}), g(\boldsymbol{s})=f(\boldsymbol{u})$.

Hence, there exists a strategy $K$-tuple $\boldsymbol{s} \in N^{g}(\boldsymbol{u})$ such that $g(\boldsymbol{s})=f(\boldsymbol{u})$.

The SCF $f$ is Nash implementable if there exists a mechanism that implements $f$ in Nash equilibria. The second condition in Definition 11 ensures that irrespective of the choice of the strategy $K$-tuple in the set $N^{g}(\boldsymbol{u})$, we always obtain the same outcome in the set of outcomes, namely $f(\boldsymbol{u})$. Such a requirement is essential for implementation, since otherwise, we would not be in a position to characterize the properties of the $\operatorname{SCF} f$. We now turn to another concept in game theory and mechanism design, namely that of strategic dominance, i.e., a particular strategy $s_{k}$ is "better" than another strategy $\hat{s}_{k}$ for a particular user $k \in$ $\mathcal{K}$, independent of the other users $j \in \mathcal{K} \backslash k$ strategies $\boldsymbol{s}_{-k}$. Even though, the concept of dominant strategy is sometimes thought of as a simplification [14], it is still a useful analytical and practical tool to investigate mechanisms and resource allocation strategies.

Definition 12. The strategy $s_{k} \in \mathcal{S}_{k}$ is a dominant strategy for user $k \in \mathcal{K}$ of $g$ at utility function $u_{k} \in \mathcal{U}_{k}$ if for all strategy $(K-1)$-tuples $\hat{\boldsymbol{s}}_{-k} \in \mathcal{S}^{K-1}, g\left(\mathbf{S}_{k}, \hat{\boldsymbol{s}}_{-k}\right) \subseteq$ $\mathbf{L}\left(g\left(s_{k}, \hat{\boldsymbol{s}}_{-k}\right), u_{k}\right)$.

Let $D S_{k}^{g}\left(u_{k}\right)$ be the set of dominant strategies for user $k$ of mechanism $g$ at utility function $u_{k}$. The strategy $K$ tuple $s \in \mathcal{S}^{K}$ is a dominant strategy equilibrium of $g$ at utility $K$-tuple $\boldsymbol{u} \in \mathcal{U}^{K}$ if for all users $k \in \mathcal{K}, s_{k} \in D S_{k}^{g}\left(u_{k}\right)$. Let $\operatorname{DS}^{g}(\boldsymbol{u})$ be the set of dominant strategy equilibria of mechanism $g$ at utility $K$-tuple $\boldsymbol{u}$.

Example 6. In the context of wireless systems, the authors of [15] show that with an appropriately designed downlink scheduler the socially optimal uplink rate allocation emerges as a dominant strategy for all users.

Definition 13. The mechanism $g$ implements the SCF $f$ in dominant strategy equilibria if for each utility $K$-tuple $\boldsymbol{u} \in \mathcal{U}^{K}$,
1. there exists a strategy $K$-tuple $\boldsymbol{s} \in D S^{g}(\boldsymbol{u})$ such that $g(\boldsymbol{s})=f(\boldsymbol{u})$ and

2. for any strategy K-tuple $\boldsymbol{s} \in D S^{g}(\boldsymbol{u}), g(\boldsymbol{s})=f(\boldsymbol{u})$.

Remark 5. The $\operatorname{SCF} f$ is dominant strategy implementable if there exists a mechanism that implements $f$ in dominant strategy equilibria.

The mechanism $g$ is called a direct revelation mechanism associated with the SCF $f$ if $\mathcal{S}=\mathcal{U}$ for all $k \in \mathcal{K}$ and $g(\boldsymbol{u})=f(\boldsymbol{u})$ for all $\boldsymbol{u} \in \mathcal{U}^{K}$. We do not distinguish between the SCF $f$ and the direct revelation mechanism associated with the $\operatorname{SCF} f$. While analyzing the implementation aspects in "Nash implementation and dominant strategy implementation" section, when we say the $\operatorname{SCF} f$, we also mean the direct revelation mechanism $g$ associated with the SCF $f$.

\section{Analysis: properties of resource allocation strategies}

For certain interference coupled wireless scenarios we would like to characterize resource allocation strategies, which satisfy certain desirable properties from the axiomatic framework, which can be implemented using mechanisms (see Figure 2).

We shall present following results in this section.

1. Results pertaining to desired properties of resource allocation strategies captured by SCFs (see sections "Nash implementation and dominant strategy implementation", "Intuitive fairness and strong intuitive fairness SCFs", and "Non-participation and continuity properties of SCFs" sections.

2. Results pertaining to Nash equilibrium implementation and Dominant strategy

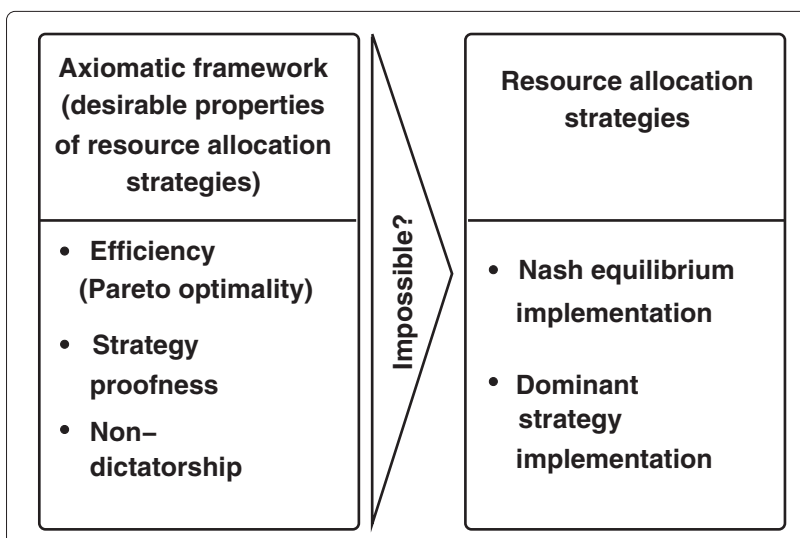

Figure 2 Abstraction: Investigation of the possibility of obtaining implementable resource allocation strategies satisfying certain desirable axioms. The left hand side of the figure displays axioms representing desirable properties of resource allocation strategies. The right hand side of the figure gives examples of possible implementation solutions. 
implementation of resource allocation strategies in a wireless network based on a SINR physical layer model (see "Nash implementation and dominant strategy implementation" section).

\section{Non-manipulable and efficient SCFs}

We begin by presenting a result, which states the following. An SCF $f$ is strategy proof, if and only if for all users $k \in \mathcal{K}$, the outcome of the resource allocation for the $k^{\text {th }}$ user, i.e., $\gamma_{k}(r)$ is a constant, which is independent of its own utility function $u_{k} \in \mathcal{U}$. However, this constant is dependent on the utility functions $u_{1}, \ldots, u_{k-1}$, $u_{k+1}, \ldots, u_{K}$, i.e., the utilities of the other users $\boldsymbol{u}_{-k}$.

Theorem 1. An SCF $f$ is strategy proof, if and only if for all users $k \in \mathcal{K}$ and for all utility function $(K-1)$-tuples $\boldsymbol{u}_{-k} \in \mathcal{U}^{K-1}$, there exists a constant $c_{k}\left(\boldsymbol{u}_{-k}\right)>0$ such that for all resource vectors $\boldsymbol{r} \in \mathbf{Q}_{k}\left(\boldsymbol{u}_{-k}\right), \gamma_{k}(\boldsymbol{r})=c_{k}\left(\boldsymbol{u}_{-k}\right)$, where $\gamma_{k}$ is the SINR function of the $k^{\text {th }}$ user.

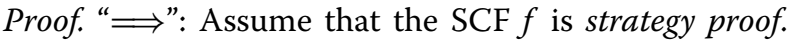
Let there be an arbitrary user $k \in \mathcal{K}$ and an utility function $(K-1)$-tuple $\boldsymbol{u}_{-k} \in \mathcal{U}^{K-1}$ also chosen arbitrarily but fixed. Then, for utility functions $u_{k}, \hat{u}_{k} \in \mathcal{U}$ chosen arbitrarily, we have that

$$
u_{k}\left(f\left(u_{k}, \boldsymbol{u}_{-k}\right)\right) \geq u_{k}\left(f\left(\hat{u}_{k}, \boldsymbol{u}_{-k}\right)\right) .
$$

Since, $\gamma_{k}$ is a special case of our utility function, the above expression follows from strategy proofness. Then $\gamma_{k}\left(f\left(u_{k}, \boldsymbol{u}_{-k}\right)\right) \geq \gamma_{k}\left(f\left(\hat{u}_{k}, \boldsymbol{u}_{-k}\right)\right)$. However, due to strategy proofness, we also have

$$
\begin{aligned}
& \hat{u}_{k}\left(f\left(\hat{u}_{k}, \boldsymbol{u}_{-k}\right)\right) \geq \hat{u}_{k}\left(f\left(u_{k}, \boldsymbol{u}_{-k}\right)\right) \\
& \gamma_{k}\left(f\left(\hat{u}_{k}, \boldsymbol{u}_{-k}\right)\right) \geq \gamma_{k}\left(f\left(u_{k}, \boldsymbol{u}_{-k}\right)\right) .
\end{aligned}
$$

Then, $\gamma_{k}\left(f\left(\hat{u}_{k}, \boldsymbol{u}_{-k}\right)\right)=\gamma_{k}\left(f\left(u_{k}, \boldsymbol{u}_{-k}\right)\right)=c_{k}\left(\boldsymbol{u}_{-k}\right)$. Since we have chosen the utility function $\hat{u}_{k} \in \mathcal{U}$ arbitrarily, we have for all resource vectors $\boldsymbol{r} \in \mathbf{Q}_{k}\left(\boldsymbol{u}_{-k}\right)$ that $\gamma_{k}(\boldsymbol{r})=$ $c_{k}\left(\boldsymbol{u}_{-k}\right)$.

“ $\Longleftarrow$ ": Let us choose a user $k \in \mathcal{K}$ arbitrarily. Let $\boldsymbol{u}_{-k} \in$ $\mathcal{U}^{K-1}$ be an arbitrarily chosen (but fixed) utility function $(K-1)$-tuple. Let $u_{k}, \hat{u}_{k} \in \mathcal{U}$ be chosen arbitrarily. Then, we have that

$$
\begin{aligned}
\gamma_{k}\left(f\left(u_{k}, \boldsymbol{u}_{-k}\right)\right) & =\gamma_{k}\left(f\left(\hat{u}_{k}, \boldsymbol{u}_{-k}\right)\right) \\
u_{k}\left(f\left(u_{k}, \boldsymbol{u}_{-k}\right)\right) & =\tilde{u}_{k}\left(\gamma_{k}\left(f\left(u_{k}, \boldsymbol{u}_{-k}\right)\right)\right) \\
& =\tilde{u}_{k}\left(\gamma_{k}\left(f\left(\hat{u}_{k}, \boldsymbol{u}_{-k}\right)\right)\right) \\
& =u_{k}\left(f\left(\hat{u}_{k}, \boldsymbol{u}_{-k}\right)\right) .
\end{aligned}
$$

Equation 10 holds for all users $k \in \mathcal{K}$. Hence, the SCF $f$ satisfies the property of strategy proofness.

We now present a result for the 2 -user case. This result shows the restriction of the available SCFs $f$, if we want them to satisfy the properties of strategy proofness and efficiency, i.e., the resource allocation strategy is nonmanipulable and is Pareto optimal.

Corollary 1. Let the number of users $K=2$. Then SCF $f$ is efficient and strategy proof, if and only if there exists a resource vector $\boldsymbol{r}^{*} \in \mathcal{R}$ with $\gamma\left(\boldsymbol{r}^{*}\right)$ a Pareto optimal resource allocation and for all utility function 2-tuples $\left(u_{1}, u_{2}\right) \in \mathcal{U}^{2}$, we have that $f\left(u_{1}, u_{2}\right)=r^{*}$.

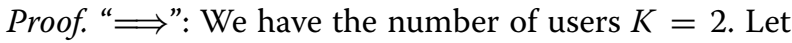
SCF $f$ be strategy proof and efficient. For $u_{2} \in \mathcal{U}\left(u_{-1}=\right.$ $u_{2}$ ) each resource $r \in \mathbf{Q}_{1}\left(u_{-1}\right)$ is on the Pareto boundary $\left(\gamma_{1}(\boldsymbol{r}), \gamma_{2}(\boldsymbol{r})\right)$ of the SINR region. From the strategy proofness of the $\operatorname{SCF} f$, for all utility functions $u_{1}, \hat{u}_{1} \in \mathcal{U}$, we have

$$
f\left(u_{1}, u_{2}\right)=f\left(\hat{u}_{1}, u_{2}\right) .
$$

Let us choose a utility function $\hat{u}_{2} \in \mathcal{U}$ for user 2 arbitrarily. Then, the following expressions hold.

$$
\begin{aligned}
& f\left(u_{1}, u_{2}\right)=f\left(u_{1}, \hat{u}_{2}\right) \\
& f\left(\hat{u}_{1}, u_{2}\right)=f\left(\hat{u}_{1}, \hat{u}_{2}\right) .
\end{aligned}
$$

Then from (11), (12), and (13) for the utility functions $u_{1}, \hat{u}_{1}, u_{2}, \hat{u}_{2}$ chosen arbitrarily, we have that $f\left(u_{1}, u_{2}\right)=$ $f\left(u_{1}, \hat{u}_{2}\right)=f\left(\hat{u}_{1}, \hat{u}_{2}\right)$. Hence, we have proved our desired result.

“ऍ": Can be easily proved.

The classical results [16,17] are for the case of pure exchange economies. Our results are for the case of beyond pure exchange economies for interference coupled systems. Theorems 1 and 1 provide certain initial intuition on the structure of strategy proof and efficient SCFs for the case of interference coupled systems. We observe that the structure imposed by the SBU function framework is quite restrictive. This structure is the basis of the impossibility results presented in Theorems $2,3,4$, and 3 .

\section{Intuitive fairness and strong intuitive fairness SCFs}

Here we present our results in relation to the restrictions obtained, when we try to obtain strategy proof and efficient resource allocation strategies, which satisfy the property of either

- intuitive fairness or

- strong intuitive fairness.

We now present a result, which states the following: a non-manipulable, efficient, and intuitive fair resource allocation strategy is independent of the downwards scaling of the utility function $u_{k} \in \mathcal{U}$ of a particular user $k \in \mathcal{K}$, when the utility function $(K-1)$-tuple $\boldsymbol{u}_{-k} \in \mathcal{U}^{K-1}$ is fixed, i.e., the resource allocation strategy is robust to 
downwards scaling of the utility function of a particular user, when the utility functions of all the other users are fixed.

Theorem 2. Let an SCF $f$ be strategy proof and efficient. Then, the SCF ffulfills the property of intuitive fair, if and only if for all users $k \in \mathcal{K}$, for all utility functions $\boldsymbol{u} \in \mathcal{U}^{K}$ and for $0<\lambda \leq 1$, we have that

$$
u_{k}\left(f\left(\lambda u_{k}, \boldsymbol{u}_{-k}\right)\right)=u_{k}\left(f\left(u_{k}, \boldsymbol{u}_{-k}\right)\right), \quad 1 \leq k \leq \mathcal{K},
$$

i.e., for $0<\lambda \leq 1$ we have that $f\left(\lambda u_{k}, \boldsymbol{u}_{-k}\right)=f\left(u_{k}, \boldsymbol{u}_{-k}\right)$.

Proof. $\Longrightarrow$ : Let SCF $f$ be strategy proof, efficient and not a constant function. Let us assume that SCF $f$ is intuitive fair. Then, we have that for all users $k \in \mathcal{K}$, for all utility function $K$-tuples $\boldsymbol{u} \in \mathcal{U}^{K}$ for $\boldsymbol{u}(\lambda)=\left(\lambda u_{k}, \boldsymbol{u}_{-k}\right), 0<\lambda \leq$ 1 and for all users $j \in \mathcal{K} \backslash k$, we have that

$$
u_{k}(f(\boldsymbol{u}(\lambda))) \geq u_{k}(f(\boldsymbol{u})) .
$$

Furthermore, we have that $\gamma_{k}(f(\boldsymbol{u}(\lambda))) \geq \gamma_{k}(f(\boldsymbol{u}))$. For user $j$ we have from Theorem 1 , that $\gamma_{k}(f(\boldsymbol{u}(\lambda)))=$ $\gamma_{k}(f(\boldsymbol{u}))$. Then, for $\boldsymbol{r}(\lambda):=f(\boldsymbol{u}(\lambda))$ we have that $u_{k}(\boldsymbol{r}(\lambda)) \geq u_{k}(f(\boldsymbol{u}))$ for $k \in \mathcal{K}$. Since, SCF $f$ is efficient, we must have that $u_{k}(\boldsymbol{r}(\lambda))=u_{k}(f(\boldsymbol{u}))$ for $k \in \mathcal{K}$.

$\Longleftarrow$ : This direction can easily be verified. Let an SCF $f$ be strategy proof, efficient and satisfy the following expression, for all users $k \in \mathcal{K}$ and $\lambda \in(0,1]$ :

$$
\begin{aligned}
u_{k}\left(f\left(\lambda u_{k}, \boldsymbol{u}_{-k}\right)\right) & =u_{k}\left(f\left(u_{k}, \boldsymbol{u}_{-k}\right)\right) \\
\text { i.e. } f\left(\lambda u_{k}, \boldsymbol{u}_{-k}\right) & =f\left(u_{k}, \boldsymbol{u}_{-k}\right) .
\end{aligned}
$$

Then, it can easily be observed that the SCF satisfies the property of intuitive fairness.

Remark 6. The SCF $f(\boldsymbol{\omega})$ defined according to (7) (in Example 7) satisfies the properties of efficiency and intuitive fairness.

We now present a corollary to Theorem 2, which states the following. Let a resource allocation strategy be nonmanipulable and efficient. If the resource allocation strategy is not robust to downward scaling of the utility function of a particular user $k \in \mathcal{K}$, then at least one other user $j \in \mathcal{K} \backslash k$ pays the price with a decrease in its performance, even if the utility functions $\boldsymbol{u}_{-k}$ are fixed.

Corollary 2. Let SCF f be strategy proof and efficient. For an arbitrarily chosen user $k \in \mathcal{K}$, with $u_{k} \in \mathcal{U}, \boldsymbol{u}_{-k} \in$ $\mathcal{U}^{K-1}$, and $\hat{\lambda} \in(0,1)$, let

$$
f\left(\hat{\lambda} u_{k}, \boldsymbol{u}_{-k}\right) \neq f\left(u_{k}, \boldsymbol{u}_{-k}\right) .
$$

Then, there exists at least one user $j \in \mathcal{K} \backslash k$ such that

$$
u_{j}\left(f\left(\hat{\lambda} u_{k}, \boldsymbol{u}_{-k}\right)\right)<u_{j}\left(f\left(u_{k}, \boldsymbol{u}_{-k}\right)\right) .
$$

Proof. Let the assumptions of the corollary be true. Let us assume that for all users $k \in \mathcal{K}$, for all utility functions $u_{k} \in \mathcal{U}$, for all utility function $(K-1)$-tuples $\boldsymbol{u}_{-k} \in \mathcal{U}^{K-1}$ and for all $j \in \mathcal{K} \backslash k$ with $\lambda \in(0,1)$ we have that

$$
u_{j}\left(f\left(\lambda u_{k}, \boldsymbol{u}_{-k}\right)\right) \geq u_{j}\left(f\left(u_{k}, \boldsymbol{u}_{-k}\right)\right) .
$$

Since, the $\operatorname{SCF} f$ satisfies the axioms of strategy proofness and efficiency, we have that

$$
u_{j}\left(f\left(\lambda u_{k}, \boldsymbol{u}_{-k}\right)\right)=u_{j}\left(f\left(u_{k}, \boldsymbol{u}_{-k}\right)\right), \quad j \in \mathcal{K} \backslash k .
$$

From Theorem 1 we have for an arbitrarily chosen user $k$, that $u_{k}\left(f\left(\lambda u_{k}, \boldsymbol{u}_{-k}\right)\right)=u_{k}\left(f\left(u_{k}, \boldsymbol{u}_{-k}\right)\right)$. Furthermore, we have that $f\left(\lambda u_{k}, \boldsymbol{u}_{-k}\right)=f\left(u_{k}, \boldsymbol{u}_{-k}\right)$ for $0<\lambda<1$.

We now present certain results, in relation to the stronger property of strong intuitive fairness.

Corollary 3. Let an SCF $f$ be strategy proof and efficient. Then, the SCF f fulfills the property of strong intuitive fair$n e s s$, if and only if for an arbitrary user $k \in \mathcal{K}$, for all $j \in \mathcal{K} \backslash k$ with utility function $(K-1)$-tuple $\boldsymbol{u}_{-k} \in \mathcal{U}^{K-1}$, there exists a constant $d_{k}\left(\boldsymbol{u}_{-k}, j\right)$ such that for all resources $\boldsymbol{r} \in \mathbf{Q}_{k}\left(\boldsymbol{u}_{-k}\right)$ we have that

$$
u_{k}(\boldsymbol{r})=d_{k}\left(\boldsymbol{u}_{-k}, j\right) \text {. }
$$

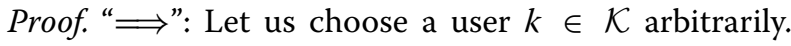
We shall take the perspective of user $k$ without any loss of generality. Let us arbitrarily choose a utility function ( $K-1)$-tuple $\boldsymbol{u}_{-k} \in \mathcal{U}^{K-1}$. We have to show that for utility functions $u_{k}, \hat{u}_{k} \in \mathcal{U}$, the expression $u_{k}\left(f\left(u_{k}, \boldsymbol{u}_{-k}\right)\right)=$ $u_{k}\left(f\left(\hat{u}_{k}, \boldsymbol{u}_{-k}\right)\right)$ holds for all $k \in \mathcal{K}$. Let us assume that there exists a user $k_{0}$, where $k_{0} \in \mathcal{K} \backslash k$, such that

$$
u_{k_{0}}\left(f\left(u_{k}, \boldsymbol{u}_{-k}\right)\right) \neq u_{k_{0}}\left(f\left(\hat{u}_{k}, \boldsymbol{u}_{-k}\right)\right) \text {. }
$$

We define $u_{k}^{*}(\boldsymbol{r})$ as follows:

$$
u_{k}^{*}(\boldsymbol{r})=\max \left(u_{k}(\boldsymbol{r}), \hat{u}_{k}(\boldsymbol{r})\right) .
$$

The utility function $u_{k}^{*}$ is strictly monotonic increasing and continuous. For all resource vectors $r \in \mathcal{R}$, we have that $u_{k}^{*}(\boldsymbol{r}) \geq u_{k}(\boldsymbol{r})$ and $u_{k}^{*}(\boldsymbol{r}) \geq \hat{u}_{k}(\boldsymbol{r})$. Therefore, from the property of intuitive fairness for all users $j \in \mathcal{K} \backslash k$, we have that

$$
\begin{array}{ll}
u_{j}\left(f\left(u_{k}^{*}, \boldsymbol{u}_{-k}\right)\right) \leq u_{j}\left(f\left(u_{k}, \boldsymbol{u}_{-k}\right)\right), & j \in \mathcal{K} \backslash k, \\
\gamma_{j}\left(f\left(u_{k}^{*}, \boldsymbol{u}_{-k}\right)\right) \leq \gamma_{j}\left(f\left(u_{k}, \boldsymbol{u}_{-k}\right)\right), & j \in \mathcal{K} \backslash k .
\end{array}
$$

From Theorem 1 we have that $\gamma_{k}\left(f\left(u_{k}^{*}, \boldsymbol{u}_{-k}\right)\right) \leq$ $\gamma_{k}\left(f\left(u_{k}, \boldsymbol{u}_{-k}\right)\right)$. Since, SCF $f$ is efficient, we must have that $\gamma_{k}\left(f\left(u_{k}^{*}, \boldsymbol{u}_{-k}\right)\right)=\gamma_{k}\left(f\left(u_{k}, \boldsymbol{u}_{-k}\right)\right)$, for all $k \in \mathcal{K}$. Therefore, from Theorem 1, we have that $f\left(u_{k}^{*}, \boldsymbol{u}_{-k}\right)=f\left(u_{k}, \boldsymbol{u}_{-k}\right)$. We can have the same expression also for $\left(u_{k}^{*}, \boldsymbol{u}_{-k}\right)$ and $\left(\hat{u}_{k}, \boldsymbol{u}_{-k}\right)$. Then, for arbitrary utility functions $u_{k}, \hat{u}_{k} \in \mathcal{U}$ we have $f\left(u_{k}, \boldsymbol{u}_{-k}\right)=f\left(\hat{u}_{k}, \boldsymbol{u}_{-k}\right)$. We have proved the desired result.

“Е": Let us choose a strategy proof and efficient SCF $f$. Let, for an arbitrary user $k \in \mathcal{K}$ and for all other users $j \in$ $\mathcal{K} \backslash k$ with utility function $(K-1)$-tuple $\boldsymbol{u}_{-k} \in \mathcal{U}^{K-1}$, there exists a constant $d_{k}\left(\boldsymbol{u}_{-k}, j\right)$ such that for each resource 
vector $\boldsymbol{r} \in \mathbf{Q}_{k}\left(\boldsymbol{u}_{-k}\right)$ we have that $u_{k}(\boldsymbol{r})=d_{k}\left(\boldsymbol{u}_{-k}, j\right)$. Then, it can easily be verified that the $\operatorname{SCF} f$ satisfies the property of strong intuitive fairness.

From the above proof, we can obtain the following additional result. If a resource allocation strategy satisfies the properties of strategy proofness, efficiency, and strong intuitive fairness, then changing the preference of a single user is not sufficient to change the resource allocation, i.e., to affect a change in the resource allocation at least two users must change their preferences or utility functions for the desired resources.

Corollary 4. Let an SCF $f$ be strategy proof and efficient. Then, the SCF ffulfills the property of strong intuitive fairness, if for all users $k \in \mathcal{K}$ and for all utility function $(K-1)$-tuples $\boldsymbol{u}_{-k} \in \mathcal{U}^{K-1}$, we have that the cardinality of the option set $\mathbf{Q}_{k}\left(u_{k}\right)$ is equal to 1 . Therefore, for any utility functions $u_{k}, \hat{u}_{k} \in \mathcal{U}$ we have $f\left(u_{k}, \boldsymbol{u}_{-k}\right)=f\left(\hat{u}_{k}, \boldsymbol{u}_{-k}\right)$.

Proof. The proof is contained in the proof of Theorem 3.

We have stated that for all utility function $K$-tuples $\boldsymbol{u}_{-k} \in \mathcal{U}^{k-1}$ and for all utility functions $u_{k} \neq 0$ and for an arbitrarily chosen user $j \in \mathcal{K} \backslash k$, we have that

$$
\inf _{r \in \mathbf{Q}_{k}\left(\boldsymbol{u}_{-k}\right)} r_{k}=\inf _{u_{k} \neq 0} f_{k}\left(\left(u_{k}, \boldsymbol{u}_{-k}\right)\right)>0 .
$$

Theorem 4 has a certain connection to the axiom nondummy introduced in [18]. An SCF $f$ is non-dummy, if $\forall k \in \mathcal{K}, \exists \boldsymbol{u} \in \mathcal{U}^{K}$ and $\hat{u}_{k} \in \mathcal{U}$, such that $f(\boldsymbol{u}) \neq f\left(\hat{u}_{k}, \boldsymbol{u}_{-k}\right)$. The non-dummy axiom states that each user can change the outcome of the SCF by changing its utility function. It guarantees every user the minimum right to affect the social decision. Then, we can say that a strategy proof, efficient, and strong intuitive fair resource allocation strategy for interference coupled systems does not satisfy the axiom non-dummy.

\section{Non-participation and continuity properties of SCFs}

In this section, we present a result, which states that if the resource allocation strategy is non-manipulable, Pareto optimal satisfies property of non-participation, then the resource allocation strategy has to be discontinuous. This has certain implications on the algorithmic implementation of resource allocation strategies. Furthermore, continuity is a desirable property for resource allocation strategies, e.g., in certain classes of widely used games, the Nash equilibrium is a continuous function of the game parameters, which follows from the implicit function theorem [19].

Theorem 3. Let an SCF $f$ be strategy proof and efficient. Then, the SCF $f$ cannot simultaneously be continuous and satisfy the property of non-participation.
Proof. Let an SCF $f$ be strategy proof and efficient. For the sake of obtaining a contradiction, let us assume that the SCF $f$ is continuous and satisfies the property of nonparticipation. Let us choose a user $k \in \mathcal{K}$ arbitrarily and take the perspective of this user $k$, without any loss in generality. Let us choose a utility function $(K-1)$ tuple $\boldsymbol{u}_{-k} \in \mathcal{U}^{K-1}$ arbitrarily. For all power vectors $\boldsymbol{p} \in$ $\mathbf{Q}_{k}\left(\boldsymbol{u}_{-k}\right)$ we have that $\gamma_{k}(\boldsymbol{p})=c_{k}\left(\boldsymbol{u}_{-k}\right)>0$. Therefore, $\frac{p_{k}}{\mathcal{I}_{k}(\boldsymbol{p})}=c_{k}\left(\boldsymbol{u}_{-k}\right)$, for all power vectors $\underline{\boldsymbol{p}} \in \mathbf{Q}_{k}\left(\boldsymbol{u}_{-k}\right)$. Exploiting the fact, that $\mathcal{I}_{k}$ is an interference function, we have that

$$
\begin{aligned}
\mathcal{I}_{k}(\underline{\boldsymbol{p}}) & =\mathcal{I}_{k}\left(\left(\boldsymbol{p}, \sigma_{k}^{2}\right)\right) \\
& \geq \mathcal{I}_{k}\left(\left(\mathbf{0}, \sigma_{k}^{2}\right)\right)=\sigma_{k}^{2} \mathcal{I}_{k}((\mathbf{0}, 1)) \\
& =\sigma_{k}^{2} \mu_{k}>0,
\end{aligned}
$$

where $0<\mu_{k}=\mathcal{I}_{k}((\mathbf{0}, 1))$. For all power vectors $\underline{\boldsymbol{p}} \in \mathbf{Q}_{k}\left(\boldsymbol{u}_{-k}\right)$ we have $c_{k}\left(\boldsymbol{u}_{-k}\right)=\frac{p_{k}}{\mathcal{I}_{k}(\underline{\boldsymbol{p}})} \leq \frac{p_{k}}{\sigma_{k}^{2} \mu_{k}}$, where $\mu_{k}=\mathcal{I}_{k}((\mathbf{0}, 1))$. Therefore, we have that the power vector $p_{k} \geq c_{k}\left(\boldsymbol{u}_{-k}\right) \sigma_{k}^{2} \lambda_{k}$, where $\lambda \in(0,1)$. Let $\boldsymbol{u}(\lambda)(\boldsymbol{p})=$ $\left(\lambda u_{k}, \boldsymbol{u}_{-k}\right)(\boldsymbol{p})$ for all $P_{\text {total }}>0$. Then, we have that

$$
\begin{gathered}
\lim _{\lambda \rightarrow 0}\left(\max _{\boldsymbol{p} \geq \mathbf{0}, \sum_{k \in \mathcal{K}} p_{k} \leq P_{\text {total }}}\left\|\boldsymbol{u}(\lambda)(\underline{\boldsymbol{p}})-\left(0, \boldsymbol{u}_{-k}\right)(\underline{\boldsymbol{p}})\right\|_{l^{1}}\right) \\
=\lim _{\lambda \rightarrow 0}\left(\operatorname{li}_{\boldsymbol{p} \geq \mathbf{0}, \sum_{k \in \mathcal{K}} p_{k} \leq P_{\text {total }}}\left|u_{k}(\underline{\boldsymbol{p}})\right|\right)=0 .
\end{gathered}
$$

Then, we have that

$$
\lim _{\lambda \rightarrow 0} f_{k}(\boldsymbol{u}(\lambda))=f_{k}\left(\left(0, \boldsymbol{u}_{-k}\right)\right)=0 .
$$

Equation 16 follows from the property of nonparticipation (Definition 8), which we have assumed that our SCF $f$ satisfies (for the sake of obtaining a contradiction). However, $f_{k}(\boldsymbol{u}(\lambda)) \geq c_{k}\left(\boldsymbol{u}_{-k}\right) \sigma_{k}^{2} \mu_{k}>0$. As can be observed that the constant $c_{k}\left(\boldsymbol{u}_{-k}\right) \sigma_{k}^{2} \mu_{k}$ is independent of $\lambda$. Therefore, $\inf _{0<\lambda<1} f_{k}(\boldsymbol{u}(\lambda))>0$, which is in contradiction with (16). Hence, we have our desired contradiction, which proves the result.

The SCF $f(\omega)$ defined in (7) (in Example 3) satisfies the properties of efficiency, continuity, and non-participation. Another practical way of justifying the non-fulfillment of the axiom of non-participation along with the other axioms in practical resource allocation strategies is that in practical resource allocation strategies a user always utilizes certain resources, i.e., in practical resource allocation strategies $f_{k}\left(0, \boldsymbol{u}_{-k}\right)=c$ for some constant $c>0$. A simple way to picture this is that even if the user decides to transmit nothing, it would require some resource to sense the channel or measure the interference temperature. Hence, the constant $c$ can be made arbitrarily small, however we always have $c>0$. 


\section{Nash implementation and dominant strategy implementation}

In this section, we present certain results pertaining to Nash equilibrium implementation and dominant strategy implementation aspects for the class of SBU functions. In this article, we have not concerned ourselves with existence and uniqueness issues of the Nash equilibrium. For the purpose of analysis of this article, we assume that these issues have been addressed. One such paper toward this direction is [20]. We begin by presenting Lemma 1, which characterizes the Nash equilibrium properties of a strategy $K$-tuple.

Lemma 1. 1. Let $\boldsymbol{u} \in \mathcal{U}^{K}$ be a fixed utility function K-tuple. Let $\boldsymbol{s} \in N^{g}(\boldsymbol{u})$ be an arbitrary strategy $K$-tuple. Then, we have for all utility function K-tuples $\hat{\boldsymbol{u}} \in \mathcal{U}^{K}$, that $\boldsymbol{s} \in N^{g}(\hat{\boldsymbol{u}})$.

2. Let $\boldsymbol{u}, \hat{\boldsymbol{u}} \in \mathcal{U}^{K}$ be arbitrary utility function K-tuples. Then, we have that $N^{g}(\boldsymbol{u})=N^{g}(\hat{\boldsymbol{u}})$.

Proof. (1) Choose an arbitrary strategy $K$-tuple $s \in$ $N^{g}(\boldsymbol{u})$. Then, we have for all users $k \in \mathcal{K}, g\left(\mathcal{S}_{k}, \boldsymbol{s}_{-k}\right) \subseteq$ $\mathbf{L}\left(g(\boldsymbol{s}), u_{k}\right)$, i.e., we have for all users $k \in \mathcal{K}$ and for all $\tilde{s}_{k} \in \mathcal{S}_{k}, q\left(\gamma_{k}\left(g\left(\tilde{s}_{k}, \boldsymbol{s}_{-k}\right)\right)\right) \leq q\left(\gamma_{k}\left(g\left(s_{k}, \boldsymbol{s}_{-k}\right)\right)\right)$, i.e., we have for all users $k \in \mathcal{K}$ and for all $\tilde{s}_{k} \in \mathcal{S}_{k}, \gamma_{k}\left(g\left(\tilde{s}_{k}, s_{-k}\right)\right) \leq$ $\gamma_{k}\left(g\left(s_{k}, s_{-k}\right)\right)$. Let $\hat{u}_{k}:=\hat{q} \cdot \gamma_{k}$, for any $k \in \mathcal{K}$. We have for all users $k \in \mathcal{K}$ and for all $\tilde{s}_{k} \in \mathcal{S}_{k}$ that $\hat{u}_{k}\left(g\left(\tilde{s}_{k}, s_{-k}\right)\right) \leq$ $\hat{u}_{k}\left(g\left(s_{k}, s_{-k}\right)\right)$, i.e., $s \in N^{g}(\hat{u})$.

(2) We need simply to exchange the order of $\hat{\boldsymbol{u}}$ and $\boldsymbol{u}$ in part (1) of the proof and we have the desired result.

A similar result as in the Nash equilibrium implementation developed in Lemma 1 can be proved for dominant strategy implementation, i.e., for arbitrary $\boldsymbol{u}, \hat{\boldsymbol{u}} \in \mathcal{U}^{K}$, we have that $D S^{g}(\boldsymbol{u})=D S^{g}(\hat{\boldsymbol{u}})$. We shall now develop the connection between the Nash equilibrium and an SCF $f$, which can be implemented in Nash equilibrium and between an SCF $f$ and its dominant strategy implementation.

Theorem 4. An SCF f can be implemented in Nash equilibrium, if and only if it is a constant function. Furthermore, an SCF $f$ can be implemented in dominant strategy, if and only if it is a constant function.

Proof. " $\Longrightarrow$ ": We shall only prove the result for the first statement of the theorem. Let $\boldsymbol{u}^{(1)}, \boldsymbol{u}^{(2)} \in \mathcal{U}^{K}$ be arbitrary utility functions in the family of functions $\mathcal{U}^{K}$, where $\boldsymbol{u}^{(1)}=\left[u_{1}^{(1)}, \ldots, u_{K}^{(1)}\right]$ and $\boldsymbol{u}^{(2)}=\left[u_{1}^{(2)}, \ldots, u_{K}^{(2)}\right]$. Let $\boldsymbol{s}^{(1)}$ and $\boldsymbol{s}^{(2)}$ be two strategy $K$-tuples such that $\boldsymbol{s}^{(1)} \in N^{g}\left(\boldsymbol{u}^{(1)}\right)$ and $\boldsymbol{s}^{(2)} \in N^{g}\left(\boldsymbol{u}^{(2)}\right)$. We have from Lemma 1 that $\boldsymbol{s}^{(1)} \in$ $N^{g}\left(\boldsymbol{u}^{(2)}\right)$. This gives us

$$
f\left(\boldsymbol{u}^{(2)}\right) \stackrel{(\mathrm{a})}{=} g\left(\boldsymbol{s}^{(2)}\right) \stackrel{(\mathrm{b})}{=} f\left(\boldsymbol{u}^{(1)}\right)
$$

Equality (a) in (17) follows from condition 2 in Definition 11 and equality (b) in (17) follows from $\boldsymbol{s}^{(1)} \in$ $N^{g}\left(\boldsymbol{u}^{(1)}\right)$.

"£": The other direction can easily be verified. The proof for the second statement of the theorem can be carried out in a similar manner.

We now compare Theorem 4 with Maskin's result in [8]. Maskin's result requires an SCF to satisfy the following two properties: monotonicity and no-veto power. In [8], an SCF $f: \mathcal{U}^{K} \mapsto \mathcal{R}$ satisfies Maskin's monotonicity condition, if $\forall \boldsymbol{r} \in \mathcal{R}$ and $\forall \boldsymbol{u}, \hat{\boldsymbol{u}} \in \mathcal{U}^{K}$, if $\boldsymbol{r}=f(\boldsymbol{u})$ and for all users $k \in \mathcal{K}, \forall \hat{\boldsymbol{r}} \in \mathcal{R}$ if $u_{k}(\boldsymbol{r}) \geq u_{k}(\hat{\boldsymbol{r}})$ implies $\hat{u}_{k}(\boldsymbol{r}) \geq \hat{u}_{k}(\hat{\boldsymbol{r}})$, then $\boldsymbol{r}=f(\hat{\boldsymbol{u}})$.

Transitioning to our case of SBU functions, let $u_{k}(\boldsymbol{r}) \geq$ $u_{k}(\hat{\boldsymbol{r}})$, for all $k \in \mathcal{K}$ and for some $\boldsymbol{u} \in \mathcal{U}^{K}$. Then, from the definition of SBU functions (Definition 1) we have $\gamma_{k}(\boldsymbol{r}) \geq$ $\gamma_{k}(\hat{\boldsymbol{r}})$, for all users $k \in \mathcal{K}$. Once again, from Definition 1 we have $\hat{u}_{k}\left(\gamma_{k}(\boldsymbol{r})\right) \geq \hat{u}_{k}\left(\gamma_{k}(\hat{\boldsymbol{r}})\right)$, for all users $k \in \mathcal{K}$ and for all $\hat{\boldsymbol{u}} \in \mathcal{U}^{K}$.

It can be observed that our class of SBU functions always satisfies the monotoncity property of Maskin. On the other hand, it does not satisfy the no-veto property (see [8], p. 31). Furthermore, we analyze a smaller class of utility functions, compared to the general class analyzed by Maskin. Therefore, the domain for our SCFs is smaller than the domain of SCFs for the results from Maskin. Hence, the class of mechanisms which can implement our SCF in Nash equilibria should potentially be larger. However, we observe from Theorem 4 that for the class of SBU functions the only permitted mechanisms, which implement the SCF in Nash equilibria or dominant strategy are constant functions.

Example 7. Consider a multiuser multiple access channel, with a beamforming array at the base station $[21,22]$. For fixed channels, the optimal beamforming weight vectors $\omega_{k}^{\text {opt }}$ for the $k$ th user, with respect to maximizing $\gamma_{k}\left(\mathbf{p}, \omega_{k}^{\mathrm{opt}}\right) \mathrm{s}$ can easily be calculated. The optimal SINR for the $k$ th user can be written as: $\gamma_{k}\left(\boldsymbol{p}, \omega_{k}^{\text {opt }}\right)=$ $p_{k} \boldsymbol{h}_{k}^{H}\left(\sigma^{2} \mathbf{I}+\sum_{j \neq k} p_{j} \boldsymbol{h}_{j} \boldsymbol{h}_{j}^{H}\right)^{-1} \boldsymbol{h}_{k}$ where $p_{k}, \boldsymbol{h}_{k}$, and $\sigma_{k}^{2}$ are the power, the channel vectors at the base station array and the noise for the $k$ th user, respectively. The interference function for the $k$ th user is, $\mathcal{I}_{k}(\boldsymbol{p})=\left(\boldsymbol{h}_{k}^{H}\left(\sigma^{2} \mathbf{I}+\right.\right.$ $\left.\left.\sum_{j \neq k, j \in \mathcal{K}} p_{j} \boldsymbol{h}_{j} \boldsymbol{h}_{j}^{H}\right)^{-1} \boldsymbol{h}_{k}\right)^{-1}$. The structure of the feasible utility region depends on several factors, for instance, the receiver strategy. For one set of beamformers $\omega_{k}$, $\forall k \in \mathcal{K}$ corresponds to one particular utility region $\mathbf{U}\left(P_{\text {total }}, \omega\right)$ for fixed channels, where $\omega=\left[\omega_{1}, \ldots, \omega_{K}\right]$ and $P_{\text {total }}$ is the total power constraint. Let a mechanism $g$ implement $f\left(\gamma_{1}^{\omega_{1}^{\mathrm{opt}}}, \ldots, \gamma_{K}^{\omega_{K}^{\mathrm{opt}}}\right)$ in Nash equilibria. Then from Theorem 4 the only permitted solution is the constant power allocation, i.e., a fixed power vector. 


\section{Literature survey}

\section{Economic literature survey}

All the work cited here is for the case of pure exchange economies. Hurwicz [23] showed that there is no strategy proof, efficient, and individually rational mechanism in 2 user 2 resource pure exchange economy. The authors of [24] attempted to replace individual rationality in Hurwicz's result with a weaker axiom of non-dictatorship. Ameliorating upon both results, Zhou [25] established an impossibility result that there is no strategy proof, efficient, and non-dictatorial mechanism in 2 user $m$ resource $(m \geq 2)$ pure exchange economies. He conjectures that there are no strategy proof, efficient, and non-inversely dictatorial mechanisms in the case of 3 or more users. In [26], Zhou's conjecture has been examined and a new class of strategy proof and efficient mechanisms in the case of four or more users (operators) was discovered.

The studies by the authors of $[18,27]$ provided examples of strategy proof, efficient, and non-dictatorial SCFs. These SCFs are also non-dummy. When we have four or more users, two-stage dictator making mechanisms are strategy proof, efficient, and non-dummy. When we have three or more users, the SCFs provided by Satterthwaite and Sonnenschein [27] are strategy proof and efficient. When we have four or more users, Kato and Ohseto [18] have shown existence of certain strategy proof, efficient, non-dummy, and dictatorial SCFs.

The property of strategy proofness requiring revealing of a users' preference is a dominant strategy. However, as can be seen from the previous results, this concept has serious drawbacks. In particular, many strategy proof mechanisms have multiple Nash equilibrium, some of which produce undesired outcomes. A possible solution to this problem is to require double implementation in Nash equilibrium and in dominant strategies. Saijo et al. [6] characterize securely implementable SCF and compare their results with dominant strategy implementations. Reichelstein and Reiter [28] discuss the realization and implementation of a social choice rule. It is shown that Nash implementation is always at least as costly, in message space size, as (decentralized) realization.

\section{Networks literature survey}

Our reference list is by no means comprehensive and the interested reader is further referred to the references in the mentioned papers. The studies of $[29,30]$ introduce the concept of a progressive second price auction. Lazar and Semret [29] have shown that a certain form of the Nash equilibrium holds when the progressive second price auction is applied by independent sellers on each link of a network with arbitrary topology. The studies of [31-33] study rules and structure of games such that their outcomes achieve certain objectives.
Huiping and Junde [34] propose a strategy proof trust management system fitting to wireless ad hoc networks. Pal and Tardos [35] have developed a general method for turning a primal-dual algorithm into a group strategy proof cost-sharing mechanism. The authors of $[36,37]$ have called nodes selfish if they are owned by independent users and their only objective is to maximize their individual goals. The article presents a game theoretic framework for truthful broadcast protocol and strategy proof pricing mechanism. Guanxiang et al. [38] have proposed an auction-based admission control and pricing mechanism for priority services, where each user pays a congestion fee for the external effect caused by their participation. The mechanism is proved to be strategy proof and efficient. Wang and $\mathrm{Li}$ [39] have addressed the issue of user cooperation in selfish and rational wireless networks using an incentive approach. They have presented a strategy proof pricing mechanism for the unicast problem. The authors of $[40,41]$ have provided a tutorial on mechanism design and attempt to apply it to concepts in engineering. Huang et al. $[42,43]$ have utilized SINR and power auctions to allocate resources in a wireless scenario and present an asynchronous distributed algorithm for updating power levels and prices to characterize convergence using supermodular game theory. Wu et al. [44] have proposed a repeated spectrum sharing game with cheatproof strategies. They propose specific cooperation rules based on maximum total throughout and proportional fairness criteria. Sharma and Teneketzis [45] have presented a decentralized algorithm to allocated transmission powers, such that the algorithm takes into account the externalities generated to the other users. Kakhbod and Teneketzis [46] consider a decentralized bandwidth/rate allocation problem in unicast service provisioning. They present a mechanism, which is implementable in Nash equilibrium, individually rational and budget balanced. Procaccia and Tennenholtz [47] advocate the reconsideration of highly structured optimization problems in the context of mechanism design. They argue that, in certain domains, approximation can be leveraged to obtain truthfulness without resorting to payments.

\section{Conclusions}

The article investigates certain desirable and natural properties of SCFs representing resource allocation strategies for interference coupled wireless systems. The property of non-manipulation and Pareto optimality of the resource allocation strategies is captured by the properties of strategy proofness and efficiency of the $S C F$, respectively. We introduce the certain desirable and natural properties of resource allocation strategies, namely (strong) intuitive fairness and non-participation. We prove that there are certain inconsistencies, among the properties of strategy proofness, efficiency, (strong) 
intuitive fairness, non-participation, and continuity. These inconsistencies result in certain limitations while having algorithmic implementations and certain analytical investigations of these resource allocation strategies. Hence, it can be observed that non-manipulation and Pareto optimality of the resource allocation strategies are stringent requirements and along with certain other desirable properties is not always implementable. It has been shown that the only permissible $S C F$ representing a resource allocation strategy in interference coupled wireless systems, which can be implemented in either Nash equilibrium or dominant strategy is the trivial constant function.

\section{Appendix}

\section{Interference functions}

Definition 14. Interference functions: We say that $\mathcal{I}$ : $\mathbb{R}_{+}^{K+1} \mapsto \mathbb{R}_{+}$is an interference function if the following axioms are fulfilled:

$A 1$ conditional positivity $\mathcal{I}(\underline{\boldsymbol{p}})>0$ if $\underline{\boldsymbol{p}}>\mathbf{0}$

$A 2$ scale invariance $\mathcal{I}(\alpha \boldsymbol{p})=\alpha \mathcal{I}(\boldsymbol{p}), \forall \alpha \in \mathbb{R}_{+}$

$A 3$ monotonicity $\mathcal{I}(\underline{\boldsymbol{p}}) \geq \mathcal{I}(\underline{\hat{\boldsymbol{p}}})$ if $\underline{\boldsymbol{p}} \geq \underline{\hat{\boldsymbol{p}}}$

$A 4$ strict monotonicity $\mathcal{I}(\underline{\boldsymbol{p}})>\mathcal{I}(\underline{\hat{\boldsymbol{p}}})$ if $\underline{\boldsymbol{p}} \geq \underline{\hat{\boldsymbol{p}}}$,

$$
\underline{p}_{K+1}>\underline{\hat{p}}_{K+1} \text {. }
$$

Note that we require that $\mathcal{I}(\boldsymbol{p})$ is strictly monotonic with respect to the last component $\underline{p}_{K+1}$. An example is $\mathcal{I}(\boldsymbol{p})=\boldsymbol{v}^{T} \boldsymbol{p}+\sigma^{2}$, where $\boldsymbol{v} \in \mathbb{R}_{+}^{K}$ is a vector of interference coupling coefficients. The axiomatic framework A1-A4 is connected with the framework of standard interference functions [2].

Definition 15. Standard interference functions: A function $Y: \mathbb{R}_{+}^{K} \mapsto \mathbb{R}_{++}$is said to be a standard interference function if the following axioms are fulfilled:

$Y 1$ positivity $Y(\boldsymbol{p})>0$, for all $\boldsymbol{p} \in \mathbb{R}_{+}^{K}$,

$Y 2$ scalability $Y(\alpha \boldsymbol{p})<\alpha Y(\boldsymbol{p})$, for all $\alpha>1$,

$Y 3$ monotonicity $Y(\boldsymbol{p}) \geq Y(\hat{\boldsymbol{p}})$ if $\boldsymbol{p} \geq \hat{\boldsymbol{p}}$.

For any constant noise power $\underline{p}_{K+1}=\sigma^{2}$ the function $Y(\boldsymbol{p})=\mathcal{I}(\boldsymbol{p})$ is standard. Conversely, any standard interference. function can be expressed within the framework A1-A4. The details about the relationship between the model A1-A4 and Yates' standard interference functions were discussed in [3] and further investigated in [48]. For the purpose of this article, it is sufficient to be aware that there exists a connection between these two models and the results of this article are applicable to standard interference functions.

\section{Competing interests}

The authors declare that they have no competing interests.

\section{Author details}

${ }^{1}$ Department of Theoretical Information Technology, Technical University of Munich, Arcisstrasse 21, 80331 Munich, Germany. ${ }^{2}$ Credit Suisse AG, Zurich, Switzerland. ${ }^{3}$ Department of Electrical and Electronic Engineering, The University of Melbourne, Parkville, VIC 3010, Australia.

Received: 16 February 2012 Accepted: 25 July 2012

Published: 7 September 2012

\section{References}

1. N Nissan, TRoughgarden, E Tardos, Algorithmic Game Theory (Cambridge University Press, Cambridge, 2007)

2. RD Yates, A framework for uplink power control in cellular radio systems. IEEE J. Sel. Areas Commun. 13(7), 1341-1348 (1995)

3. M Schubert, $\mathrm{H}$ Boche, QoS-based resource allocation and transceiver optimization. Found. Trends Commun. Inf. Theory. 2 (2006). doi:10.1561/0100000010

4. R Johari, JN Tsitsiklis, Efficiency of scalar-parameterized mechanisms. Oper. Res. 57(4), 823-839 (2009)

5. DNC Tse, SV Hanly, Linear multiuser receivers: effective interference, effective bandwidth and user capacity. IEEE Trans. Inf. Theory. 45(2), 641-657 (1999)

6. T Saijo, T Sjöström, T Yamato, Secure implementation. Theor. Econ. 2, 203-229 (2007)

7. A Mas-Colell, MD Whinston, JR Green, Microeconomic Theory (Oxford University Press, New York, 1995)

8. E Maskin, Nash equilibrium and welfare optimality. Rev. Econ. Stud. 66 23-38 (1999)

9. S Babera, B Peleg, Strategy-proof voting schemes with continuous preferences. Social Choice Welfare. 7, 31-38 (1990)

10. HJM Peters, Axiomatic Bargaining Game Theory (Kluwer Academic Publishers, Dordrecht, 1992)

11. W Thomson, The fair division of a fixed supply among a growing population. Math. Oper. Res. 8, 319-326 (1983)

12. J Mo, J Walrand, Fair end-to-end window-based congestion control. IEEE/ACM Trans. Netw. 5(8), 556-567 (2000)

13. L Massoulie, J Roberts, Bandwidth sharing: objectives and algorithms. IEEE/ACM Trans. Netw. 3(10), 320-328 (2002)

14. KS Chung, JC Ely, Foundations of dominant strategy mechanisms. Robust Mechanism Design (Cowles Foundation for Research in Economics, Yale University, 2004)

15. J Price, $T$ Javidi, Leveraging downlink for efficient uplink allocation in a single-hop wireless network. IEEE Trans. Inf. Theory. 53(11), 4330-4339 (2007)

16. A Gibbard, Manipulation of voting schemes: a general result. Econometrica. 41, 587-601 (1973)

17. MA Satterthwaite, Strategy-proofness and arrow's conditions: existence and correspondence theorems for voting procedures and social welfare functions. J. Econ. Theory. 10(2), 187-217 (1975)

18. M Kato, S Ohseto, Non-dummy agents in pure exchange economies. Jpn. Econ. Rev. 55(2), 212-220 (2004)

19. T Alpcan, Noncooperative games for control of networked systems. PhD thesis, University of Illinois at Urbana-Champaign, Urbana, IL, 2006

20. S Naik, T Alpcan, $\mathrm{H}$ Boche, in IEEE ICC, A Nash equilibrium analysis of interference coupled wireless systems, Cape Town, South Africa, 2010

21. M Schubert, H Boche, in International Zurich Seminar on Broadband Communications, A unifying theory for uplink and downlink multi-user beamforming, 2002, pp. 27-1-27-5

22. S Stanczak, H Boche, M Wiczanowski, in IEEE WCNC, Towards better understanding of medium access control for multiuser beamforming systems, 2005, pp. 689-694

23. L Hurwicz, Decision and Organization, chap. on Informationally Decentralized Systems (North-Holland, Amsterdam, 1972), pp. 297-336

24. P Dasgupta, P Hammmond, E Maskin, The implementation of social choice rules: some general results of incentive Compatibility. Rev. Econ. Stud. 46, 185-216 (1979)

25. L Zhou, Inefficiency of strategy-proof allocation mechanisms in pure exchange economies. Social Choice Welfare. 8, 247-254 (1991)

26. M Kato, S Ohseto, Towards general impossibility theorems in pure exchange economies. Social Choice Welfare. 19, 659-664 (2002) 
27. MA Satterthwaite, $\mathrm{H}$ Sonnenschein, Strategy-proof allocation mechanisms at differntiable points. Rev. Econ. Stud. 48, 587-597 (1981)

28. S Reichelstein, $\mathrm{S}$ Reiter, Game forms with minimal strategy spaces. Econometrica. 49, 661-692 (1988)

29. AA Lazar, N Semret, in International Symposium on Dynamic Games and Applications, The progressive second price auction mechanism for network resource sharing, Maastricht, Netherlands, 1998

30. P Jia, PE Caines, in Proceedings of International Conference on Game Theory for Networks (GameNets2009), Equilibria and convergence of auctions on networks, Istanbul, Turkey, 2009, pp. 268-276

31. S Yang, B Hajek, VCG-Kelly mechanisms for allocation of divisible goods: adapting VCG mechanisms to one-dimensional signals. IEEE J. Sel. Areas Commun. 25(6), 1237-1243 (2007)

32. RT Maheswaran, T Basar, in 43rd IEEE Conference on Decision and Control $(C D C)$, Social welfare of selfish agents: motivating efficiency for divisible resources, vol. 2, 2004, pp. 1550-1555

33. A Dimakis, R Jain, J Walrand, in 45th IEEE Conference on Decision and Control (CDC), Mechanisms for efficient allocation in divisible capacity networks, San Diego, CA, USA, 2006

34. S Huiping, $S$ Junde, in Canadian Conference on Electrical and Computer Engineering, 2004, Strategyproof trust management in wireless ad hoc network, vol. 3, 2004, pp. 1593-1596

35. M Pal, E Tardos, in Proceedings of the 44th Annual IEEE Symposium on Foundations of Computer Science, 2003, Group strategy proof mechanisms via primal-dual algorithms, 2003 pp. 584-593

36. NR Suri, in IEEE International Confernce on Computer Communications (INFOCOM), Design of incentive compatible protocols for wireless networks: a game theoretic approach, 2006, pp. 1-2

37. NR Suri, Y Narahari, D Manjunath, in First International Conference on Communication System Software and Middleware, 2006. Comsware 2006, An efficient pricing based protocol for broadcasting in wireless ad hoc networks, 2006, pp. 1-7

38. Z Guanxiang, L Yan, Y Zongkai, C Wenqing, in 29th Annual IEEE International Conference on Local Computer Networks, 2004, Auction-based admission control and pricing for priority services, 2004, pp. 398-399

39. WZ Wang, XY Li, in Proceedings of the 18th International Parallel and Distributed Processing Symposium, 2004, Truthful low-cost unicast in selfish wireless networks, 2004, p. 219

40. D Garg, Y Narahari, S Gujar, Foundations of mechanism design: a tutorial, Part 1-key concepts and classical results. Sadhana. 33(3), 83-130 (2008)

41. D Garg, Y Narahari, S Gujar, Foundations of mechanism design: a tutorial. Part 2-advanced concepts and results. Sadhana. 33(2), 131-174 (2008)

42. J Huang, R Berry, M Honig, Distributed interference compensation for wireless networks. IEEE J. Sel. Areas Commun. 24(5), 1074-1084 (2006)

43. J Huang, R Berry, M Honig, Auction-based spectrum sharing. ACM Mob. Netw. Appl. J. 24(5), 405-418 (2006)

44. Y Wu, B Wang, KJR Liu, TC Clancy, Repeated open spectrum sharing game with cheat-proof strategies. IEEE Trans. Wirel. Commun. 8(4), 1922-1933 (2009)

45. S Sharma, D Teneketzis, An Externalities-Based Decentralized Optimal Power Allocation Algorithm for Wireless Networks. IEEE/ACM Trans. on Networking. 17(6), 1819-1831 (2009)

46. A Kakhbod, D Teneketzis, in Proceedings of the 47th annual Allerton conference on Communication, control, and computing, An individually rational, budget-balanced mechanism that implements in Nash equilibria the solution of the unicast service provisioning problem, 2009

47. AD Procaccia, M Tennenholtz, in Proceedings of the 10th ACM Conference on Electronic Commerce, Approximate mechanism design without money, Stanford, CA, USA, 2009

48. H Boche, M Schubert, in IEEE International Conference on Communications (ICC), Unified framework for interference modeling of multi-user wireless networks, Dresden, Germany, 2009

doi:10.1186/1687-1499-2012-284

Cite this article as: Boche et al:: Mechanism design and implementation theoretic perspective for interference coupled wireless systems. EURASIP Journal on Wireless Communications and Networking 2012 2012:284. 